\title{
Spectroscopic analysis of LiTmF4
}

\section{Christensen, H.P.}

\section{Published in:}

Physical Review B

Link to article, DOI:

10.1103/PhysRevB.19.6573

Publication date:

1979

\section{Document Version}

Publisher's PDF, also known as Version of record

Link back to DTU Orbit

Citation (APA):

Christensen, H. P. (1979). Spectroscopic analysis of LiTmF4. Physical Review B, 19(12), 6573-6582. https://doi.org/10.1103/PhysRevB.19.6573

\section{General rights}

Copyright and moral rights for the publications made accessible in the public portal are retained by the authors and/or other copyright owners and it is a condition of accessing publications that users recognise and abide by the legal requirements associated with these rights.

- Users may download and print one copy of any publication from the public portal for the purpose of private study or research.

- You may not further distribute the material or use it for any profit-making activity or commercial gain

- You may freely distribute the URL identifying the publication in the public portal

If you believe that this document breaches copyright please contact us providing details, and we will remove access to the work immediately and investigate your claim 


\title{
Spectroscopic analysis of $\mathrm{LiTmF}_{4}$
}

\author{
H. P. Christensen \\ Department of Electrophysics, The Technical University of Denmark, DK-2800 Lyngby, Denmark
}

(Received 12 February 1979)

\begin{abstract}
The absorption spectra of $\mathrm{Tm}^{3+}$ in $\mathrm{LiTmF}_{4}$ have been measured at $2,10,30$, and $50 \mathrm{~K}$ in the spectral interval $4000-25000 \mathrm{~cm}^{-1}$. The energy levels of the ground-state configuration were calculated by diagonalizing the Hamiltonian of the electron-electron interaction, the spin-orbit coupling, and the crystal field in a basis of the whole configuration. The electrostatic parameter $F_{2}$, the spin-orbit parameter $\zeta$, and the crystal-field parameters $B_{k q}$ were varied to obtain the best agreement with the experimentally observed levels. As the model does not account for configuration mixing and minor magnetic effects, it was necessary after optimizing $F_{2}$ and $\zeta$ to match the centers of gravity for the multiplets before the final adjustment of the $B$ parameters. When this was done, the standard deviation was lowered from 170 to $12 \mathrm{~cm}^{-1}$. The $B$ parameters obtained for $\mathrm{Tm}^{3+}$ have been compared to those of $\mathrm{Tb}^{3+}, \mathrm{Ho}^{3+}$, and $\mathrm{Er}^{3+}$ in $\mathrm{LiLnF}_{4}$, and they follow a common trend. The intensities of the transitions from the ground state were calculated in the JuddOfelt scheme, fitting six complex intensity parameters $A(k q \lambda)$ for best agreement with the experimentally observed intensities. The model was only able to give a rough estimate of the transition probabilities. The obtained relative standard deviation was 1.1. Contrary to what was found in the case of the energy calculations, it was important for the intensity calculations that the $B$ parameters were allowed to take complex values. The imaginary part of the $A$ parameters was not important to the intensities.
\end{abstract}

\section{INTRODUCTION}

This paper is the third and last in a series of papers reporting on spectroscopic investigation of dense $\mathrm{LiLnF}_{4}$ crystals, where $\mathrm{Ln}$ - which is tripositive in $\mathrm{LiLnF}_{4}$ - is one of the heavier elements of the lanthanide group (the first series of rare earths).

The first paper was concerned with $\mathrm{LiTbF}_{4}{ }^{1}$ the second with $\mathrm{LiHoF}_{4}$ and $\mathrm{LiErF}_{4},{ }^{2}$ and here the results for $\mathrm{LiTmF}_{4}$ are given. Spectroscopic data for $\mathrm{Tm}^{3+}$ diluted in $\mathrm{LiYF}_{4}$ have been reported by Jenssen et $a l{ }^{3}$

The chief purpose of this series of papers has been the experimental determination of the energy levels and the extraction of the crystal-field parameters for the Ln site in $\mathrm{LiLnF}_{4}$. In order to exploit the information in the experimental data better, for $\mathrm{LiTmF}_{4}$ it has also been tried to estimate the intensities of the optical transitions and compare them with those experimentally observed.

The theory for the energy and intensity calculations is summed up in Sec. II. The experimental data are presented in Sec. III, and the calculated values are given in Sec. IV. In Sec. V the results are discussed in connection with those obtained in the two preceeding papers.

\section{THEORY}

Going up through the heavy $\mathrm{Ln}^{3+}$ ions, the ground-state configuration becomes increasingly simple and separated from excited configurations.
The ground configuration of $\mathrm{Tm}^{3+}$ is $4 f^{12}$, which is 91 times degenerate. The ground multiplet is ${ }^{3} H_{6}$. Apart from the isolated ${ }^{1} S$ level, which is situated around $78000 \mathrm{~cm}^{-1}$ above the ground level, the extension of the ground configuration is approximately $39000 \mathrm{~cm}^{-1}$. The distance from the ground level to the lowest level of the next configuration is approximately 60000 $\mathrm{cm}^{-1}\left(\right.$ Dieke $\left.^{4}\right)$.

\section{A. Energy levels}

The Hamiltonian for the system is given by

$$
H=H_{\mathrm{ee}}+H_{\mathrm{so}}+H_{\mathrm{cf}},
$$

where

$$
H_{\mathrm{ee}}=e^{2} \sum_{i>j} \frac{1}{r_{i j}}
$$

is the electron-electron interaction,

$$
H_{\mathrm{so}}=\zeta \sum_{i}\left(\overrightarrow{\mathrm{I}}_{i} \cdot \overrightarrow{\mathrm{s}}_{i}\right)
$$

is the spin-orbit coupling, and

$$
H_{\mathrm{cf}}=\sum_{k q} B_{k q} \sum_{i}\left(C_{i}\right)_{q}^{(k)}
$$

is the crystal field. The $B$ parameters are given by

$$
B_{k q}=\left\langle r^{k}\right\rangle A_{k q} \text {. }
$$

The expansion of the crystal field in terms of Legendre polynomials (which are equivalent to the 
$C$ operators) is only a formal way of introducing the symmetry of the crystal, since it has not been possible to set up a model for calculating the $A$ parameters.

The matrix elements of $H_{\text {ee }}$ may be expressed as linear combinations of the radial parameters $F_{2}$, $F_{4}$, and $F_{6}$. For $f$ electrons it is a good approximation to set $F_{4}=0.138 F_{2}$ and $F_{6}=0.0151 F_{2}$. Hence, there are only two free-ion parameters: $F_{2}$ and the spin-orbit parameter $\zeta$.

For Ln ions heavier than Sm, LiLnF $\mathrm{F}_{4}$ crystallizes in the stable scheelite structure (space group $\left.I 4_{1} / a\right)$. At the Ln site the point symmetry is $S_{4}$. In this symmetry the only nonvanishing crystal-field parameters with $k$ even influencing $f$ electrons are $A_{20}, A_{40}, A_{44}, A_{60}$, and $A_{64}$, where the coordinate system may be chosen, so only the imaginary part of $A_{64}$ differs from zero. The parameters with $k$ odd give no contribution to the crystal-field splitting of the multiplets within a single configuration.

In $S_{4}$ symmetry the multiplets split up in nondegenerate levels, whose eigenfunctions transform according to the $\Gamma_{1}$ or $\Gamma_{2}$ representation of the point group and in two times degenerate levels transforming according to $\Gamma_{3} \Gamma_{4}$ (in the notation of Koster et al. ${ }^{5}$ ).

\section{B. Transition probabilities}

Electric-dipole (ED) transitions are to first order forbidden within a single configuration, since the ED operator is of odd parity. The magnetic-dipole (MD) operator is of even parity, but other selection rules restrict MD transitions to occur between multiplets for which $J$ does not differ by more than one.

For Ln ions at sites without inversion symmetry (as in $S_{4}$ symmetry), transitions within the ground configuration are seen between most of the levels without the restriction $\Delta J \leqq 1$. An explanation of these transitions were given by $\mathrm{Judd}^{6}$ and Ofelt, ${ }^{7}$ and the details for the present case will be sketched in the following.

\section{Intensity model}

Sites without inversion symmetry possess crystalfield terms with $k$ odd, and these mix configurations of opposite parity into the ground configuration. ED transitions are now allowed between the new levels with mixed wave functions, which do not have a definite parity.

The ED operator is given by

$$
\overrightarrow{\mathrm{H}}_{\mathrm{ed}}=-e \sum_{i} \overrightarrow{\mathrm{r}}_{i} \text {. }
$$

The integrated intensity $I$ of a transition between two states $\Psi_{1}$ and $\Psi_{2}$ is given by

$$
I=c \Delta E \sum_{p}\left|\left\langle\Psi_{1}\left|\left(H_{I}\right)_{p}^{(1)}\right| \Psi_{2}\right\rangle\right|^{2},
$$

where $c$ is a constant, $\Delta E$ is the energy difference between the levels, and $\left(H_{\mathrm{ed}}\right)_{p}^{(1)}$ is the $p$ th component of the transition operator on tensor form. For ED transitions $p=0$ are related to $\pi$ polarization and $|p|=1$ to $\sigma$ polarization. The matrix elements $H_{12}^{p}$ of $\left(H_{\mathrm{ed}}\right)_{p}^{(1)}$ between two mixed states $\psi_{1}^{\prime}$ and $\psi_{2}^{\prime}$ are given by the expression

$$
\begin{aligned}
H_{12}^{p}=\sum_{i} & {\left[\left\langle\psi_{1}\left|\left(H_{\mathrm{ed}}\right)_{p}^{(1)}\right| \phi_{i}\right\rangle\left\langle\phi_{i}\left|H_{\mathrm{cf}}\right| \psi_{2}\right\rangle /\left(E_{2}-E_{i}\right)\right.} \\
& \left.+\left\langle\psi_{1}\left|H_{\mathrm{cf}}\right| \phi_{i}\right\rangle\left\langle\phi_{i}\left|\left(H_{\mathrm{ed}}\right)_{p}^{(1)}\right| \psi_{2}\right\rangle /\left(E_{1}-E_{i}\right)\right],
\end{aligned}
$$

where $\psi_{1}$ and $\psi_{2}$ are the pure wave functions of the ground configuration, and $\phi_{i}$ are wave functions of excited configurations of opposite parity. $E_{1}$, $E_{2}$, and $E_{i}$ are the energies of the corresponding levels.

In the Judd-Ofelt model it is now assumed that $\left|E_{1}-E_{2}\right| \ll\left|E_{1}-E_{i}\right|$, i.e., $E_{2} \simeq E_{1}$, and that the excited configurations are completely degenerate. These approximations are unfortunately not always well fulfilled. After some algebraic manipulations one then arrives at the expression

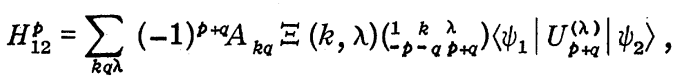

where the two last factors in the terms are a $3-j$ symbol and a matrix element of a unit tensor operator, respectively. For $f$ electrons $\Xi(k, \lambda)$ is given by

$$
\begin{gathered}
\Xi(k, \lambda)=2 \sum_{n l}(-1)^{k+i}[f][l][\lambda] \frac{1}{E_{1}-E_{n l}}\left(\begin{array}{c}
f 1 l l \\
000
\end{array}\right)\left(\begin{array}{c}
f k l \\
000
\end{array}\right) \\
\times\langle 4 f|r| n l\rangle\left\langle 4 f\left|\gamma^{k}\right| n l\right\rangle\left\{\begin{array}{l}
1 k \lambda \\
f f l
\end{array}\right\},
\end{gathered}
$$

where $[x]=2 x+1, f=3$, and the last factor in the terms is a $6-j$ symbol. The sum runs over excited configurations $\phi$ of the form $4 f^{11} n l$, where $l=f \pm 1$.

\section{Intensity parameters}

It is not possible to make correct $a b$ initio calculations of $\Xi(k, \lambda)$, since it requires the knowledge of the energies and the radial part of the wave functions of excited configurations of parity opposite to that of the ground configuration.

One possibility for obtaining values for the $\Xi(k, \lambda)$ parameters is to exclude in the calculations all configurations but the few lowest. This approximation, however, is not good. Even if the mixing from the lower configurations is much stronger than from the higher, the density of the configurations grows so fast, as the continuum is approached, that the total effect from these configu- 
rations is not negligible.

Another possibility is to treat $\Xi(k, \lambda)$ as adjustable parameters. For $f$ electrons $k$ may take the odd values 3,5 , and 7 , and $\lambda$ the values 2,4 , and 6 . A triangle rule on the $6-j$ symbol limits the nonvanishing parameters to $\Xi(3,2), \Xi(3,4), \Xi(5,4)$, $\Xi(5,6)$, and $\Xi(7,6)$. In $S_{4}$ symmetry the crystalfield parameters with $k$ odd are $A_{32}, A_{52}, A_{72}$, and $A_{76}$, which may all be complex. As was the case for the even crystal-field parameters, the odd parameters cannot be calculated from first principles. Hence, the crystal-field parameters must also be treated as variable parameters.

To limit the number of free parameters, the $\Xi(k, \lambda)$ and $A_{k q}$ parameters may be combined to new parameters:

$$
A(k q \lambda)=A_{k q} \Xi(k, \lambda) \text {. }
$$

This gives six complex parameters: $A(322)$, $A$ (324), $A(524), A(526), A(726)$, and $A$ (766). There are, however, restrictions on some of these parameters:

$$
\begin{aligned}
& \operatorname{Im} A(324)=\operatorname{Im} A(322)[\operatorname{Re} A(324) / \operatorname{Re} A(322)], \\
& \operatorname{Im} A(526)=\operatorname{Im} A(524)[\operatorname{Re} A(526) / \operatorname{Re} A(524)],
\end{aligned}
$$

which reduce the number of free parameters to ten. According to Minhas and Sharma, ${ }^{8}$ further restrictions are valid for these parameters: $\operatorname{Re} A$ (324) must have the same sign as $\operatorname{Re} A(322)$, and $\operatorname{Re} A(526)$ the same sign as $\operatorname{Re} A(524)$.

\section{EXPERIMENTS}

\section{A. Experimental setup}

A single crystal of LiTm $\mathrm{L}_{4}$ was oriented by $\mathrm{x}$-ray technique, and a slab about $1 \mathrm{~mm}$ thick was cut with faces perpendicular to an $a$ axis. Examination of the slab revealed some small blisters or grains in the crystal.

The absorption spectra were recorded using a modified Zeiss MM12 monochromator. A Glan prism was used as polarizer. The spectral resolution was better than $6 \mathrm{~cm}^{-1}$. For low-temperature

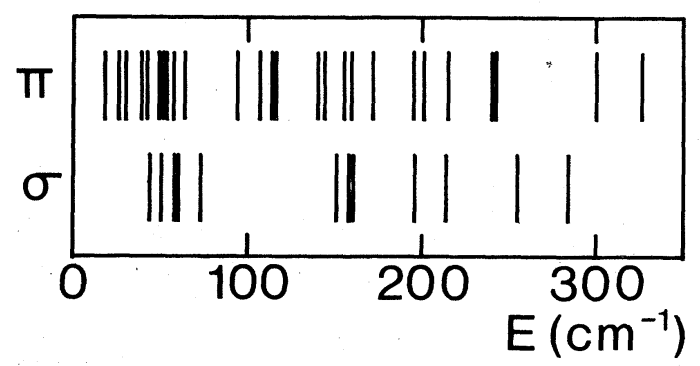

FIG. 1. Distance in energy at $2 \mathrm{~K}$ from the zero-phonon lines to the lines of the sidebands. measurements a simple glass-cryostat system was used, as described elsewhere. ${ }^{9}$

\section{B. Experimental results}

The absorption spectra for polarized light were recorded for $\mathrm{LiTmF}_{4}$ in the region from 4000 to $25000 \mathrm{~cm}^{-1}(2.5-0.4 \mu \mathrm{m})$ at $2,10,30$, and $50 \mathrm{~K}$. This spectral interval covers the transitions from the ground multiplet to the other multiplets of the three lowest terms $\left({ }^{3} H,{ }^{3} F\right.$, and $\left.{ }^{1} G\right)$.

\section{Interpretation of the lines}

At $2 \mathrm{~K}$ only transitions from the ground state may be seen. The wave function of the ground state transforms according to the $\Gamma_{2}$ representation of $S_{4} . \Gamma_{2} \rightarrow \Gamma_{1}$ ED transitions occur in $\pi$ polarization, $\Gamma_{2} \rightarrow \Gamma_{3} \Gamma_{4}$ in $\sigma$ polarization, whereas $\Gamma_{2} \rightarrow \Gamma_{2}$ transitions of ED nature are not allowed.

Thus at $2 \mathrm{~K}$ only lines corresponding to transitions to $\Gamma_{1}$ and $\Gamma_{3} \Gamma_{4}$ levels should occur. Actually, more lines were seen, but many of them were weak and broad. The broad lines were always situated at the high-energy side of strong and rather sharp lines. All the sharp lines could be interpreted as ED transitions to $\Gamma_{1}$ or $\Gamma_{3} \Gamma_{4}$ levels. A few of the expected lines were missing.

The lines of the sidebands must be due to phononassisted transitions. The experimental fact that the side lines always had higher energy than the zero-phonon line-i.e., phonons are emitted in the processes - is in accordance with the fact that at $2 \mathrm{~K}$ there are no phonons to be absorbed. A collection of energy differences from the sharp lines to the broad lines are plotted in Fig. 1.

The energies of the phonon modes in $\mathrm{LiYF}_{4}$ are given by Miller et al.,${ }^{10}$ and the energies of the phonon modes in $\mathrm{LiTmF}_{4}$ should probably not deviate much from these. Although it looks like the energy differences in Fig. 1 are grouped around certain values, there is no similarity to the energy scheme for the phonon modes in $\mathrm{LiYF}_{4}$. The previous examinations of $\mathrm{LiLnF}_{4}$ crystals $^{1,2}$ have also shown that the regular phonon modes in these crystals normally are rather inactive. The sidebands in $\mathrm{LiTmF}_{4}$ may therefore involve local phonons due to lattice defects.

In order to determine the energy of the levels to which transitions were not seen at $2 \mathrm{~K}$, the spectra were recorded at $10 \mathrm{~K}, 30 \mathrm{~K}$, and $50 \mathrm{~K}$. The warm-up spectra were consistent with additional transitions from a $\Gamma_{3} \Gamma_{4}$ level at $32 \mathrm{~cm}^{-1}$ and a $\Gamma_{1}$ level at $57 \mathrm{~cm}^{-1}$.

To examine the temperature dependence of the transitions, the transitions to the $\Gamma_{3} \Gamma_{4}$ level at $21207 \mathrm{~cm}^{-1}$ were followed to room temperature. The line position and linewidth are shown as a 
TABLE I. Experimental and calculated energies in $\mathrm{cm}^{-1}$ and calculated term composition of the wave functions for the levels of the lowest multiplets of $\mathrm{Tm}^{3+}$ in $\mathrm{LiTmF}_{4} . \Delta c$ gs gives the adjustment of the calculated centers of gravity for the multiplets. Experimental and calculated intensities of the transitions from the ground level to the other levels. " 0 " in the column of Expt. intens. indicates that the transition was not seen at $2 \mathrm{~K}$, and " $\infty$ " that the absorption was too strong to be determined with the sample used.

\begin{tabular}{|c|c|c|c|c|c|c|c|c|}
\hline$J$ & $\Gamma$ & $\begin{array}{c}\text { Expt. } \\
\text { energy }\end{array}$ & $\begin{array}{c}\text { Calc. } \\
\text { energy }\end{array}$ & $\Delta c g s$ & $\begin{array}{l}\text { Wave } \\
\text { funct. }\end{array}$ & pol. & $\begin{array}{c}\text { Expt. } \\
\text { intens. }\end{array}$ & $\begin{array}{l}\text { Calc. } \\
\text { intens. }\end{array}$ \\
\hline \multirow[t]{8}{*}{6} & 2 & 0 & 0 & \multirow[t]{10}{*}{$\cdots$} & $99 \%^{3} \mathrm{H}$ & $\ldots$ & $\ldots$ & $\ldots$ \\
\hline & 34 & 32 & 31 & & $1 \%^{1} I$ & ... & $\cdots$ & 0.2 \\
\hline & 1 & 57 & 62 & & & $\ldots$ & ... & 0.1 \\
\hline & 2 & ... & 287 & & & ... & $\ldots$ & 0 \\
\hline & 2 & $\ldots$ & 316 & & & ... & $\ldots$ & 0 \\
\hline & 1 & $\ldots$ & 365 & & & $\ldots$ & ... & 6 \\
\hline & 34 & $\ldots$ & 382 & & & $\cdots$ & $\ldots$ & 51 \\
\hline & 34 & $\cdots$ & 410 & & & $\ldots$ & $\ldots$ & 106 \\
\hline & 1 & $\ldots$ & 421 & & & $\ldots$ & $\ldots$ & 1 \\
\hline & 2 & $\cdots$ & 436 & & & $\cdots$ & $\ldots$ & 0 \\
\hline \multirow[t]{7}{*}{4} & 1 & 5585 & 5570 & \multirow[t]{7}{*}{97} & $57 \%^{3} \mathrm{~F}$ & $\pi$ & 1 & 4 \\
\hline & 34 & 5757 & 5746 & & $34 \%^{1} G$ & $\sigma$ & 86 & 62 \\
\hline & 1 & 5757 & 5762 & & $9 \%{ }^{3} \mathrm{H}$ & $\pi$ & 35 & 94 \\
\hline & 2 & 5828 & 5822 & & & $\ldots$ & 0 & 0 \\
\hline & 1 & 5957 & 5976 & & & $\pi$ & 29 & 19 \\
\hline & 2 & 5961 & 5955 & & & $\ldots$ & 0 & 0 \\
\hline & 34 & 5965 & 5983 & & & $\sigma$ & $\infty$ & 922 \\
\hline \multirow[t]{8}{*}{5} & 2 & 8270 & 8263 & \multirow[t]{8}{*}{-138} & $99 \%{ }^{3} H$ & $\cdots$ & 0 & 0 \\
\hline & 34 & 8287 & 8278 & & $+{ }^{3} F,{ }^{1} G$ & $\sigma$ & 34 & 23 \\
\hline & 1 & 8287 & 8296 & & & $\pi$ & 30 & 76 \\
\hline & 34 & 8500 & 8490 & & & $\sigma$ & 97 & 77 \\
\hline & 1 & 8519 & 8532 & & & $\pi$ & 61 & 1 \\
\hline & 2 & $\cdots$ & 8533 & & & $\ldots$ & 0 & 0 \\
\hline & 34 & $\cdots$ & 8541 & & & ... & 0 & 14 \\
\hline & 1 & $\cdots$ & 8555 & & & $\cdots$ & 0 & 158 \\
\hline \multirow[t]{7}{*}{4} & 2 & 12595 & 12597 & \multirow[t]{7}{*}{-130} & $58 \%{ }^{3} H$ & $\ldots$ & 0 & 0 \\
\hline & 1 & 12615 & 12616 & & $29 \%^{3} F$ & $\pi$ & 5 & 21 \\
\hline & 34 & 12650 & 12636 & & $13 \%^{1} G$ & $\sigma$ & 54 & 76 \\
\hline & 1 & 12740 & 12742 & & & $\pi$ & $\infty$ & 29 \\
\hline & 1 & 12805 & 12802 & & & $\pi$ & 28 & 13 \\
\hline & 34 & 12830 & 12839 & & & $\sigma$ & 37 & 195 \\
\hline & 2 & $\cdots$ & 12913 & & & $\cdots$ & 0 & 0 \\
\hline \multirow[t]{5}{*}{3} & 34 & 14540 & 14531 & \multirow[t]{5}{*}{-269} & $99 \%{ }^{3} \mathrm{~F}$ & $\sigma$ & 31 & 35 \\
\hline & 2 & 14557 & 14560 & & $+{ }^{3} H,{ }^{1} G$ & $\ldots$ & 0 & 0 \\
\hline & 34 & 14600 & 14609 & & & $\sigma$ & 34 & 37 \\
\hline & 2 & ... & 14609 & & & ... & 0 & 0 \\
\hline & 1 & 14625 & 14620 & & & $\pi$ & $\infty$ & 856 \\
\hline \multirow[t]{4}{*}{2} & 2 & 15092 & 15096 & \multirow[t]{4}{*}{-332} & $78 \%{ }^{3} \mathrm{~F}$ & $\ldots$ & 0 & 0 \\
\hline & 34 & 15210 & 15194 & & $19 \%{ }^{1} D$ & $\sigma$ & 31 & 38 \\
\hline & $\begin{array}{r}04 \\
2\end{array}$ & 10210 & 15218 & & $2 \%^{3} P$ & $\ldots$ & $\begin{array}{r}01 \\
0\end{array}$ & $\begin{array}{r}00 \\
0\end{array}$ \\
\hline & 1 & 15255 & 15267 & & $+{ }^{3} H,{ }^{1} G$ & $\ldots$ & 0 & 4 \\
\hline \multirow[t]{7}{*}{4} & 1 & 20980 & 20999 & \multirow[t]{7}{*}{102} & $53 \%{ }^{1} G$ & $\pi$ & 1 & 1 \\
\hline & 34 & 21207 & 21207 & & $37 \%{ }^{3} \mathrm{H}$ & $\sigma$ & 61 & 45 \\
\hline & 2 & 21297 & 21297 & & $10 \%^{3} F$ & $\ldots$ & 0 & 0 \\
\hline & 1 & 21320 & 21334 & & & $\pi$ & 134 & 39 \\
\hline & 2 & 21526 & 21513 & & & ... & 0 & 0 \\
\hline & 34 & 21575 & 21573 & & & $\sigma$ & $\infty$ & 411 \\
\hline & 1 & 21578 & 21556 & & & $\pi$ & 21 & 6 \\
\hline
\end{tabular}

function of the temperature in Fig. 2. It is seen that within the experimental accuracy the line position is temperature independent. The broadening with increasing temperature is moderate.

\section{Line intensity}

The transmission coefficient $T(E)$ for light with photon energy $E$ through a crystal is approximately given by 


$$
T(E) \simeq(1-R) e^{-\alpha(E) d},
$$

where $R$ is the reflection coefficient, $\alpha(E)$ is the loss coefficient of the crystal, and $d$ is the thickness of the crystal. For energies which are equal to the difference between two energy levels, $\int \alpha(E) d E$ is approximately proportional to the intensity $I$ [Eq. (7)] of the transition between these levels, if the lowest is completely populated.

If reflection is neglected, i.e., if the transmission coefficient between the lines is set equal to.1, the intensity of a transition may be deter mined from the experimental transmission in the following way:

$$
I=\frac{c^{\prime}}{d} \int \ln \frac{1}{T(E)} d E
$$

where $c^{\prime}$ is a constant.

For weak absorption $(T \simeq 1) \ln [1 / T(E)] \simeq 1-T(E)$, which means that

$$
I \simeq \frac{c^{\prime}}{d} \int[1-T(E)] d E
$$

may be used for crystals with weak absorption, e.g., lightly doped crystals. For dense crystals this approximation is not valid. The intensity of the strong transitions would be estimated too low. Most important is that since $\ln [1 / T(E)]$ tends towards infinity for $T(E)$ going towards zero, it is not possible to determine the intensity of lines with absorption near $100 \%$.

The experimental intensities of the transitions at $2 \mathrm{~K}$ (for $c^{\prime} / d \equiv 1 \mathrm{~cm}^{-1}$ ) are listed in Table I.
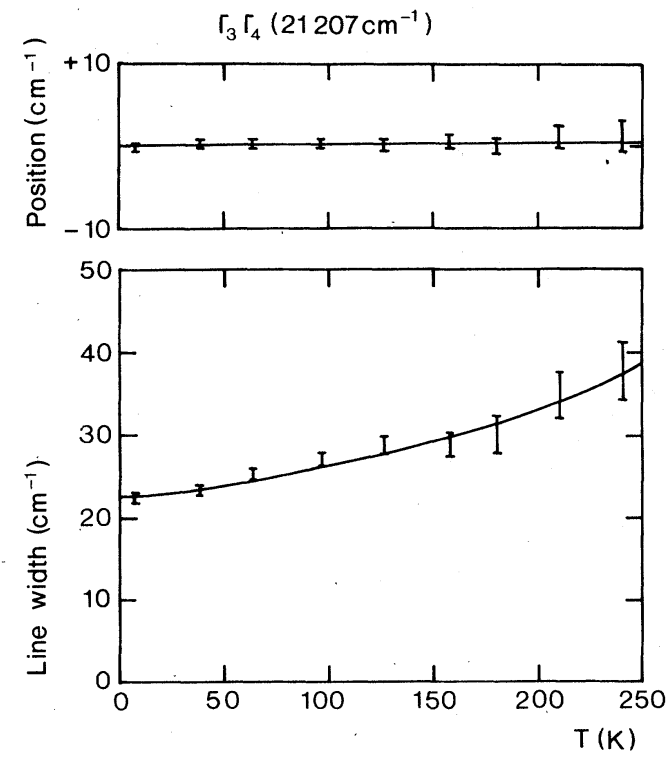

FIG. 2. Line position and linewidth of the transition from the ground level to the $\Gamma_{3} \Gamma_{4}$ level at $21207 \mathrm{~cm}^{-1}$ of $\mathrm{Tm}^{3+}$ in $\mathrm{LiTmF}_{4}$ as a function of temperature.

\section{CALCULATiONS}

The three lowest terms of $4 f^{12}$ are heavily mixed via the $J=4$ multiplets. This can be seen from the free-ion correlation diagram given in Fig. 3, in which the energies of the multiplets are plotted as function of the ratio of the spin-orbit parameter $\zeta$ to the electrostatic parameter $F_{2}$. In this diagram pure multiplets will show up as straight lines. Due to the strong term mixing calculations within the ground term are of no use for $\mathrm{Tm}^{3+}$. The Hamiltonian has to be diagonalized in a larger basis.

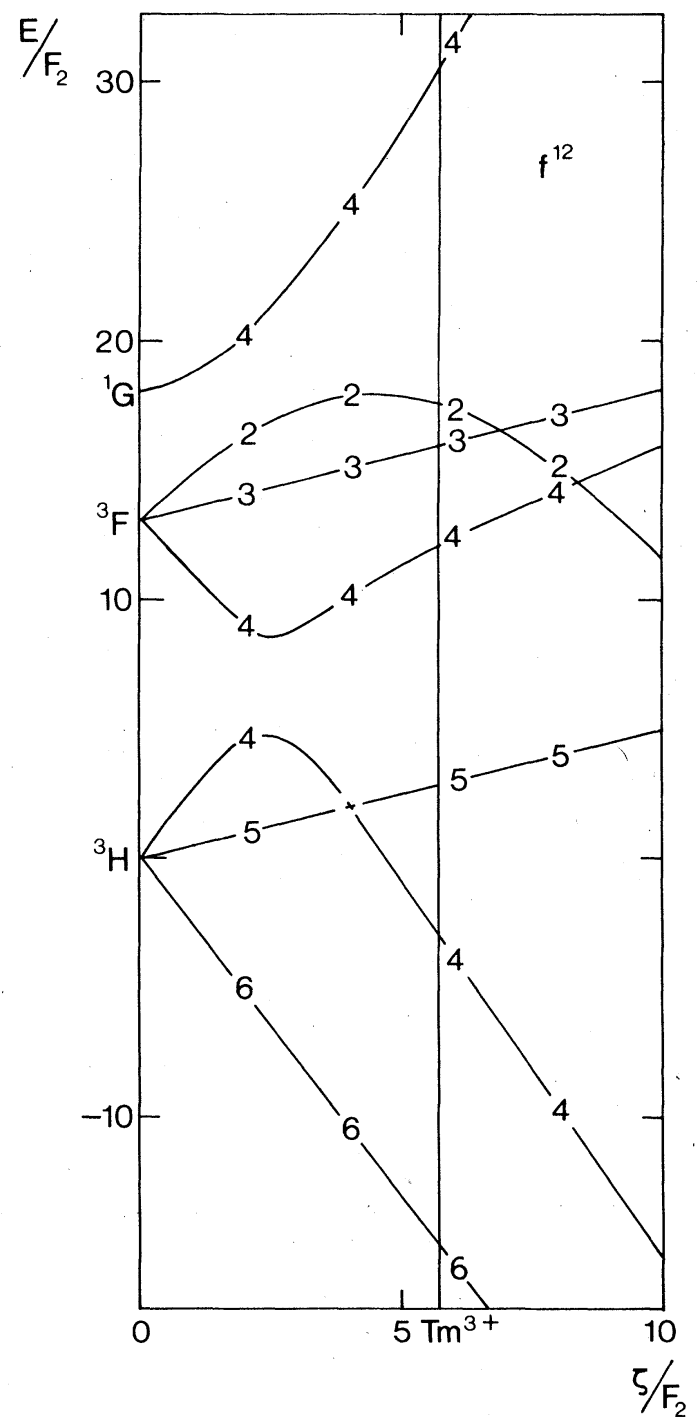

FIG. 3. Energy-normalized with respect to $F_{2}$-of multiplets of free ions with $f^{12}$ configuration as a function of the ratio of the spin-orbit parameter $\zeta$ to the electrostatic parameter $F_{2}$. The multiplets are identified by their $J$ number. The position of $\mathrm{Tm}^{3+}$ is indicated by the vertical line. 


\section{A. Energy levels}

The energy levels of the ground configuration were calculated by diagonalizing the energy Hamiltonian described in Sec. II A in a $L S$ basis of the configuration. The $F_{2}$ parameter, the $\zeta$ parameter, and the six $B$ parameters were varied until the best agreement was obtained with the $35 \mathrm{ex}-$ perimentally observed levels listed in Table I.

The standard deviation $s$ of the energy fit is given by

$$
s=\left(\sum_{i=1}^{N} \frac{\left(\Delta E_{i}\right)^{2}}{N-M}\right)^{1 / 2},
$$

where $N$ is the number of levels, and $M$ is the number of free parameters.

The fitting procedure gave an $s$ of $170 \mathrm{~cm}^{-1}$. A better agreement was not to be expected, since no attempt has been made to account for configuration mixing. If this mixing is omitted, it is not possible in the calculations to arrive at the right centers of gravity for the multiplets.

The standard deviation of $170 \mathrm{~cm}^{-1}$ was obtained with the $F_{2}$ and $\zeta$ values listed in Table II together with the free-ion values given by Wybourne. ${ }^{11}$ It is important that the fitted values do not differ too much from the free-ion values - otherwise the model is unrealistic.

The way the configuration mixing is normally introduced in the calculations (see, e.g., Ref. 3) is not easily seen through, and may as well account for other inadequacies of the model. Instead of modifying the model, it was decided to fix $F_{2}$ and $\zeta$ on the values obtained, match the centers of gravity for each multiplet, and vary the $B$ parameters only. In this way an $s$ of $12 \mathrm{~cm}^{-1}$ was obtained. The $B$ parameters of this fit are given in the second column of Table III.

The calculated energy levels and term composition of the wave functions are listed in Table I. The term composition of the wave functions do not differ from level to level within a multiplet. The adjustment of the center of gravity for each multiplet is also given in Table I.

TABLE II. Electron-electron and spin-orbit parameters in $\mathrm{cm}^{-1}$ for $\mathrm{Tm}^{3+}$.

\begin{tabular}{lcc}
\hline \hline & In $\operatorname{LiTmF}_{4}$ & Free ion \\
\hline$F_{2}$ & 469 & 451 \\
$F_{4}$ & $(64.7)^{\mathrm{a}}$ & 68.1 \\
$F_{6}$ & $(7.1)^{\mathrm{a}}$ & 7.4 \\
$\zeta$ & 2663 & 2656 \\
\hline
\end{tabular}

${ }^{a}$ Not allowed to vary freely.
Setting $\operatorname{Im} B_{64}$ equal to zero and varying the five remaining $B$ parameters only, gave an agreement just as good as when all six parameters were varied. The parameters obtained in this way are listed in the first column of Table III. The observation that $\operatorname{Im} B_{64}$ has little influence on the energies of the levels is in agreement with the fact that on the Ln site in the scheelite structure the local symmetry is almost $D_{2 d}$, in which all parameters are real. Although the wave functions are real in $D_{2 d}$ symmetry, their term composition does not differ from that of the complex $S_{4}$ wave function within the accuracy of the calculations.

\section{B. Transition probabilities}

The intensities of the ED transitions from the ground state to the other levels of the ground configuration were calculated using the model described in Sec. IIB1. The energies and the wave functions of the levels used in these calculations were those obtained from fitting to the energy levels. The intensities of the MD transitions were not considered, since they are only allowed to the ${ }^{3} \mathrm{H}_{5}$ multiplet, and there was no experimental evidence for their presence. This is in contrast to the case of $\mathrm{LiHoF}_{4}$ and $\mathrm{LiErF}_{4}{ }^{2}$ where MD transitions to the first excited multiplet were comparable in strength to the ED transitions.

The ten $A$ parameters of Sec. II B 2 were varied until the best agreement with the experimental data was obtained. Of the 23 transitions observed at $2 \mathrm{~K}$, only 19 were used in the fitting procedure, since four were so strong that it was not possible to get a realistic value for the intensity with the thickness of the sample used. As a measure of the discrepancy between experimental and calculated intensities the relative standard deviation $\sigma$ was used. $\sigma$ is given by

$$
\sigma=\left(\sum_{i=1}^{N} \frac{\left[2\left(I_{i}^{\mathrm{cal}}-I_{i}^{\mathrm{ex}}\right) /\left(I_{i}^{\mathrm{cal}}+I_{i}^{\mathrm{ex}}\right)\right]^{2}}{N-M}\right)^{1 / 2},
$$

where $N$ is the number of transitions, and $M$ is the number of free parameters. The reason for

TABLE III. Crystal-field parameters in $\mathrm{cm}^{-1}$ for $\mathrm{Tm}^{3+}$ in $\mathrm{LiTmF}_{4}$. (i) $\operatorname{Im} B_{64} \equiv 0$; (ii) $\operatorname{Im} B_{64}$ allowed to vary freely; (iii) $\operatorname{Im} S_{64} \equiv 320 \mathrm{~cm}^{-1}$. The uncertainty on the parameters are $\pm 10 \mathrm{~cm}^{-1}$.

\begin{tabular}{lrrr}
\hline & i & ii & \multicolumn{1}{c}{ iii } \\
\hline$B_{20}$ & 368 & 367 & 364 \\
$B_{40}$ & -717 & -716 & -711 \\
$B_{44}$ & 919 & 918 & 884 \\
$B_{60}$ & -65 & -64 & -64 \\
$\operatorname{Re} B_{64}$ & 619 & 615 & 594 \\
$\left|\operatorname{Im} B_{64}\right|$ & 0 & 118 & 320 \\
\hline \hline
\end{tabular}


using the relative standard deviation is to give all the transitions the same weight in the fitting procedure. Minhas and Sharma, ${ }^{8}$ who use the absolute deviation, fit actually to the strongest transitions only.

Contrary to what was the case for the energy fits, it was not possible to get rapid convergency towards a minimum for the intensity fits. It was difficult to find good starting values for the $A$ parameters. Neither the parameters given for $\mathrm{Nd}^{3+}$ in $\mathrm{PbMiO}_{4}$ by Minhas and Sharma, ${ }^{8}$ nor the parameters which could be calculated from the theoretical works of Morrison et al. ${ }^{12}$ and Wortman et $a l .{ }^{13}$ gave good results. The best convergence was obtained by starting with all the $A$ parameters set equal to zero.

As stated in Sec. IV A, it is a usable approximation in the energy calculations to assume $D_{2 d}$ symmetry. In this symmetry $\Gamma_{1}$ of $S_{4}$ splits up in two different representations. In $S_{4}$ symmetry transitions from the ground state are allowed to both types of levels, whereas only transitions to one of the types are allowed in $D_{2 d}$ symmetry. Experimentally transitions to both types of levels are observed, even if many of the $D_{2 d}$ forbidden lines are weak. Therefore, it is not possible to use $D_{2 d}$ symmetry in the intensity calculations.

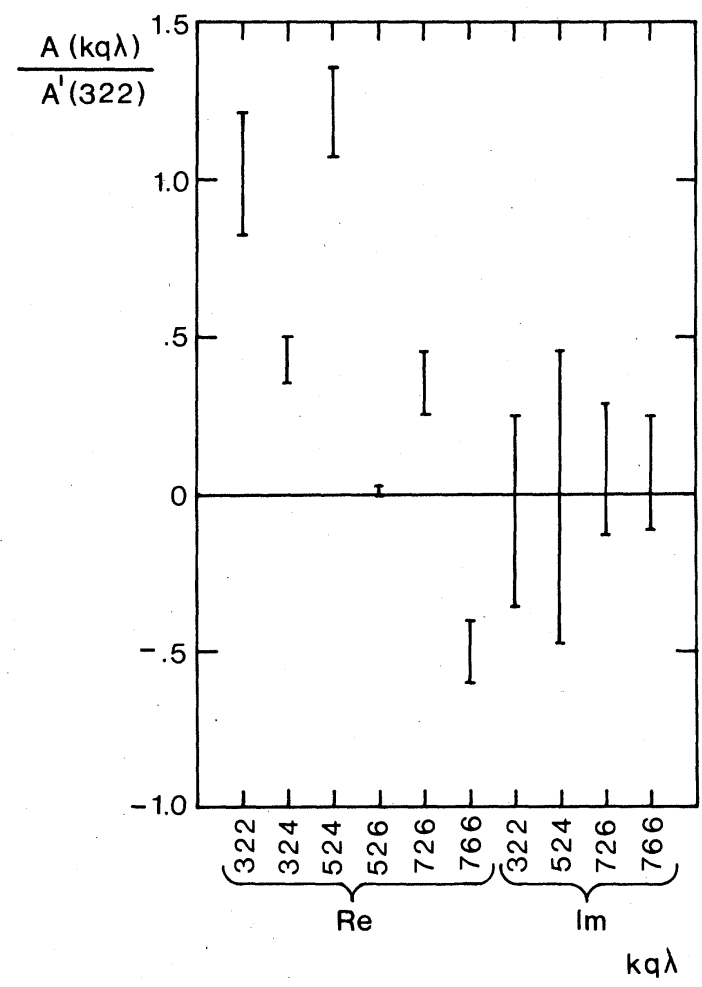

FIG. 4. Range in which the relative parameters $A(k q \lambda)$ may run without altering the relative standard deviation more than $10 \%$.
Since it is not possible to get a good determination of $\operatorname{Im} B_{64}$ from the energy fit, the intensity fits might be used to estimate the value of this parameter. The dependence of the intensities on this parameter was rather strong and peculiar. There was indeed a minimum in $\sigma$ around the value $118 \mathrm{~cm}^{-1}$ obtained from the energy fit, but there was another and deeper minimum around $320 \mathrm{~cm}^{-1}$. The minima were separated by a high sharp peak in the proximity of $200 \mathrm{~cm}^{-1}$.

With $\operatorname{Im} B_{64}$ fixed on $320 \mathrm{~cm}^{-1}$, a new energy fit was carried out. The agreement obtained was as good as in the two previous fits with $\operatorname{Im} B_{64}$ equal to zero or to $118 \mathrm{~cm}^{-1}$, respectively. The new $B$ parameters are listed in the last column of Table III. It is seen that changes in $\operatorname{Im} B_{64}$ mostly affect the other nondiagonal parameters, $B_{44}$ and $\operatorname{Re} B_{64}$.

It is not possible to determine the $\operatorname{sign}$ of $\operatorname{Im} B_{64}$, neither in the energy fit nor in the intensity fit. But the signs of the imaginary parts of the $A$ parameters depend on the sign of $\operatorname{Im} B_{64}$.

With the $B$ parameters of the third column of Table III an intensity fit was obtained with $\sigma=1.1$. The calculated intensities are given in Table I and the corresponding values of the $A$ parameters normalized with respect to the real part of $A(322)$ are presented in the second column of Table IV. The absolute values of the $A$ parameters have no meaning, since the parameters are fitted to relative intensities. The signs of the imaginary parts of the $A$ parameters are those obtained for $\operatorname{Im} B_{64} \equiv+320 \mathrm{~cm}^{-1}$.

The minimum of $\sigma$ was not very sharp. In Fig. 4 the intervals in which each parameter may run without altering the relative standard deviation more than $10 \%$ are shown. It is seen that the imaginary parts of the parameters are very badly determined; they may run over both positive and negative values. With all the imaginary parts of the $A$ parameters set equal to zero the real parameters listed in the first column of Table IV were

TABLE IV. Intensity parameters for $\mathrm{Tm}^{3+}$ in $\mathrm{LiTmF}_{4}$. The parameters are normalized with respect to $\operatorname{Re} A(322) . \quad A(k q \lambda)=A_{k q} \Xi(k \lambda)$, where $A_{k q}$ are crystal-field parameters and $\Xi(k \lambda)$ are parameters involving radial integrals.

\begin{tabular}{lll}
\hline & Real & Complex \\
\hline$A(322)$ & 1 & $1 \quad+i 0.16$ \\
$A(324)$ & 0.45 & $0.46(+i 0.07)^{\mathrm{a}}$ \\
$A(524)$ & 1.12 & $1.16+i 0.36$ \\
$A(526)$ & 0.02 & $0.00(+i 0.00)^{\mathrm{a}}$ \\
$A(726)$ & 0.45 & $0.30+i 0.24$ \\
$A(766)$ & -0.50 & $-0.55-i 0.06$ \\
\hline
\end{tabular}

${ }^{a}$ Not allowed to vary freely. 
obtained. The agreement was as good as when all the parameters were varied ( $\sigma$ was lowered to 0.9 due to the fewer variable parameters). That the imaginary parts of the $A$ parameters have little influence on the intensities in the scheelite structure is in contrast to the conclusion of Minhas and Sharma. ${ }^{8}$

\section{DISCUSSION}

\section{A. Energy levels}

The difference between the experimental energy levels of $\mathrm{Tm}^{3+}$ in $\mathrm{LiTmF}_{4}$ and in $\mathrm{LiYF}_{4}$ (Ref. 3) are less than $20 \mathrm{~cm}^{-1}$, which may partly be due to experimental inaccuracy. There is no systematic change in the crystal-field splittings of the multiplets when going from one host to the other. Thus there cannot be any substantial difference between the crystal-field potentials at the Ln site in these two crystals.

Jenssen et al. ${ }^{3}$ have calculated the energy levels of the ground configuration of $\mathrm{Tm}^{3+}$ in $\mathrm{LiYF}_{4}$. They use a more complicated Hamiltonian than the one described in Sec. II A, but their agreement with the experimental levels is only slightly better than the agreement obtained in the present work. Refinements of the model do not have much influence on the splittings of the multiplets.

The crystal-field parameters for $\mathrm{Tm}^{3+}$ in $\mathrm{LiTmF}_{4}$ are shown in Fig. 5 together with the parameters for $\mathrm{Tb}^{3+}, \mathrm{Ho}^{3+}$, and $\mathrm{Er}^{3+}$ in $\mathrm{LiLnF}_{4}{ }^{1,2}$ To indicate the variation through the series of $\mathrm{Ln}^{3+}$ ions, parabolas are drawn through the values for $\mathrm{Tb}^{3+}$, $\mathrm{Ho}^{3+}$, and $\mathrm{Tm}^{3+}$. The values for $\mathrm{Er}^{3+}$ are not used in fixing these parabolas due to the difficulties in determining the parameters for this ion as described in Ref. 2. The points for $\mathrm{Er}^{3+}$ are, however, close to the parabolas. The values for $\mathrm{Tb}^{3+}, \mathrm{Ho}^{3+}$, and $\mathrm{Er}^{3+}$ are obtained by fitting to levels of the ground term only, whereas the values for $\mathrm{Tm}^{3+}$ are obtained by fitting to levels of the three lowest terms. The dependence of the parameters on the collection of levels to which they are fitted is, however, weak, if the levels under consideration possess correct wave functions (see Ref. 2).

Considering the uncertainties on the parameters, the parabolas should not be considered as more than a hint to the sizes of the parameters for the remaining ions. A further uncertainty is the possible temperature dependence of the parameters. Contrary to what was found for $\operatorname{LiTmF}_{4}$, the parameters for $\mathrm{LiTbF}_{4}$ (Ref. 1) were temperature dependent. (The values given for $\mathrm{Tb}^{3+}$ in Fig. 5 are for $T=10 \mathrm{~K}$.)

If the extrapolated values for $\mathrm{Eu}^{3+}$ should turn out to be realistic, the crystal-field potential in $\mathrm{LiEuF}_{4}$ would differ rather much from what is found in other scheelites. Taken together with the observed temperature-dependent crystal field in $\mathrm{LiTbF}_{4}$, one might speculate if the $\mathrm{LiLnF}_{4}$ structure becomes increasingly unstable when going from $\mathrm{LiTmF}_{4}$ to $\mathrm{LiEuF}_{4}$ (in accordance with the

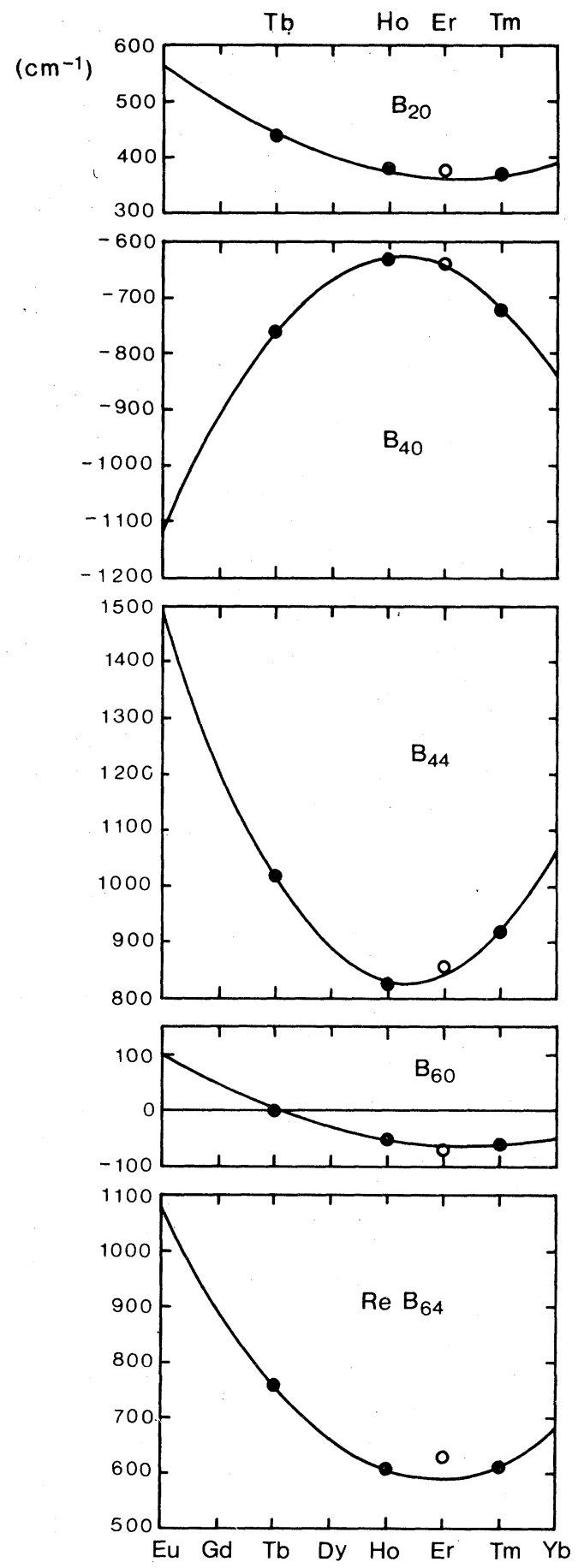

FIG. 5. Real crystal-field parameters at $10 \mathrm{~K}$ for $\mathrm{Tb}^{3+}, \mathrm{Ho}^{3+}, \mathrm{Er}^{3+}$, and $\mathrm{Tm}^{3+}$ in $\mathrm{LiLnF}_{4}$. Parabolas are drawn through the values for $\mathrm{Tb}^{3+}, \mathrm{Ho}^{3+}$, and $\mathrm{Tm}^{3+}$. 
circumstance that $\mathrm{LiSm}_{4}$ should not exist $\left.{ }^{14}\right)$. The spectra of $\mathrm{LiEuF}_{4}$ would be interesting to investigate $\left(\mathrm{LiGdF}_{4}\right.$ have no transitions in the optical region), but we have not succeeded in growing crystals of $\mathrm{LiEuF}_{4}$ by the method of spontaneous crystallization as described by Laursen and Holmes. ${ }^{15}$

Karayianis et al.$^{16}$ give crystal-field parameters for $\mathrm{Nd}^{3+}, \mathrm{Ho}^{3+}, \mathrm{Er}^{3+}$, and $\mathrm{Tm}^{3+}$ in $\mathrm{LiYF}_{4}$. The values for $\mathrm{Ho}^{3+}$ are in good agreement with those obtained in $\mathrm{LiHoF}_{4}$. For $\mathrm{Er}^{3+}$ and $\mathrm{Tm}^{3+}$ the parameters differ up to $150 \mathrm{~cm}^{-1}$. There are, however, no systematic change, so for the heavy Ln ions the crystal field does not seem to vary much from dense to diluted crystals; a statement in agreement with the conclusion for $\mathrm{Tm}^{3+}$ in the beginning of this section. Most of the parameters for $\mathrm{Nd}^{3+}$ follow the trends given by the parabolas in Fig. 2, but their numerical values are much smaller than extrapolations from the curves would give.

The calculated term composition of the wave functions showed-as could be predicted from Fig. 3-that multiplets with equal $J$ quantum numbers were heavily mixed. The mixing of multiplets with different $J$ values - which can take place only due to the crystal field-was very weak. This shows that it would be a good approximation to consider the crystal field as a perturbation within each multiplet, when the wave functions of these were determined. Since the wave functions of the ground multiplet of $\mathrm{Tm}^{3+}$ (due to the great distance to other multiplets with $J=6$ ) are almost pure ${ }^{3} H$, it should for this ionin contrast to what was the case for $\mathrm{Tb}^{3+}$ (Ref.1) be a rather good approximation to do calculations only within the ground multiplet. The choice of basis set in a given situation may differ from one $\mathrm{Ln}$ ion to another because the relative strength of the three terms in Eq. (1) changes through the series of Lanthanides.

It must be emphasized that the wave functions belonging to a branch in the diagram of Fig. 3 outgoing from a given term do not necessarily maintain to be dominated by that term. The lowest multiplet with $J=4$ originates from ${ }^{3} H$, but the composition of the wave functions are for $\mathrm{Tm}^{3+}$ mostly ${ }^{3} F$. Hence the multiplet could equally well be named ${ }^{3} H_{4}$ or ${ }^{3} F_{4}$.

\section{B. Transition probabilities}

The relative experimental intensities for $\mathrm{Tm}^{3+}$ in $\mathrm{LiYF}_{4}$ (Ref. 3) differed somewhat from those

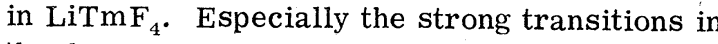
the dense crystal were relatively weaker in the diluted crystal.

The intensity calculations gave only a rough estimate of the strength of the transitions. Except for a few lines the model was capable to point out the.very strong and very weak lines. Even if the parameters were varied over a wide range, the model seldom failed in selecting strong and weak transitions. The intensity model was, however, not able to give quantitative good line intensities. The $A$ parameters must only be considered as fitting parameters. As well as including the effect of configuration mixing due to the static crystal field, the parameters more or less include the effects of lattice vibrations.

As a minor point it should be mentioned that the intensity calculations indicated that the interpretation of the $\Gamma_{1}$ levels of the ${ }^{3} H_{5}$ multiplet was somewhat ambiguous. The experimental $\pi$ line at $8519 \mathrm{~cm}^{-1}$ was interpreted as the $\Gamma_{1}$ line calculated to $8532 \mathrm{~cm}^{-1}$. This line, however, had a very low calculated intensity, whereas the line calculated to $8555 \mathrm{~cm}^{-1}$ had high intensity, and experimentally there was seen no $\pi$ line with higher energy. If the experimental line at 8519 $\mathrm{cm}^{-1}$ instead was interpreted as the $8555 \mathrm{~cm}^{-1}$ line, it was possible to obtain an energy fit with an $s$ of $14 \mathrm{~cm}^{-1}$. However, when the modification did not improve the energy fit, it is unsafe to make a line assignment on the basis of the intensity calculations.

The theoretical intensities calculated by Wortman et al. ${ }^{13}$ for $\mathrm{Tm}^{3+}$ in $\mathrm{LiYF}_{4}$ were not in agreement with the intensities for $\mathrm{Tm}^{3+}$ in $\mathrm{LiTmF}_{4}$. Especially (and in contrast to the calculations reported here) the agreement was bad for $\sigma$ transitions. Nor were the theoretical results in agreement with the experimental intensities for $\mathrm{Tm}^{3+}$ in $\mathrm{LiYF}_{4},{ }^{3}$ although the disagreement for $\sigma$ polarization was less than when compared to the results for the dense crystal.

\section{ACKNOWLEDGMENTS}

The author is very grateful to Dr. R. Nevald and Professor V. Frank for helpful suggestions and discussions during the preparation of the manuscript. 
${ }^{1}$ H. P. Christensen, Phys. Rev. B 17, 4060 (1978).

${ }^{2}$ H. P. Christensen, Phys. Rev. B 19 , 6564 (1979).

${ }^{3}$ H. P. Jenssen, A. Linz, R. P. Leavitt, C. A. Morrison, and D. E. Wortman, Phys. Rev. B 11, 92 (1975).

${ }^{4} \mathrm{G}$. H. Dieke, Spectra and Energy Levels of Rare-Earth Ions in Crystals (Wiley, New York, 1969).

${ }^{5}$ G. F. Koster, J. O. Dimmock, R. G. Wheeler, and $\mathrm{H}$. Statz, Properties of the Thirty-Two Point Groups (MIT, Cambridge, Mass., 1963).

${ }^{6}$ B. R. Judd, Phys. Rev. 127, 750 (1962).

${ }^{7}$ G. S. Ofelt, J. Chem. Phys. 37, 511 (1962).

${ }^{8}$ I. S. Minhas and Kailash K. Sharma, Phys. Rev. B 14, 4124 (1976).

${ }^{9}$ H. P. Christensen, J. Phys. E 11,742 (1978).

${ }^{10}$ S. A. Miller, H. E. Rost, and $\bar{H}$. H. Caspers, J. Chem.
Phys. 52, 4172 (1970).

${ }^{11} \mathrm{~B}$. G. Wyborne, Spectroscopic Properties of the Rare Earth (Interscience, New York, 1965).

${ }^{12}$ C. A. Morrison, N. Karayianis, and D. E. Wortman, Harry Diamond Laboratories Report No. TR-1816, 1977 (unpublished).

${ }^{13}$ D. E. Wortman, N. Karayianis, and C. A. Morrison, Harry Diamond Laboratories Report No. TR-1770, 1976 (unpublished).

${ }^{14}$ R. E. Thoma, G. D. Brunton, R. A. Penneman, and T. K. Keenan, Inorg. Chem. 9, 1096 (1970).

${ }^{15}$ I. Laursen and L. M. Holmes, J. Phys. C ㄱ, 3765 (1974).

${ }^{16}$ N. Karayianis, D. E. Wortman, and H. P. Jenssen, J. Phys. Chem. Solids 47, 675 (1976). 